\title{
A New Type of Internal Hernia After Laparoscopic Roux-en-Y Gastric Bypass
}

\author{
A. Paroz • J. M. Calmes $\cdot$ S. Romy $\cdot$ V. Giusti $\cdot$ M. Suter
}

Received: 16 October 2008 / Accepted: 4 November 2008 /Published online: 26 November 2008

(C) Springer Science + Business Media, LLC 2008

\begin{abstract}
Roux-en-Y gastric bypass (RYGBP) is currently the most common bariatric procedure. One of its late complications is the development of internal hernia, which can lead to acute intestinal obstruction or recurrent colicky abdominal pain. The aim of this paper is to present a new, unusual, and so far not reported type of internal hernia. A common computerized database is maintained for all patients undergoing bariatric surgery in our departments. The charts of patients with the diagnosis of internal hernia were reviewed. Three patients were identified who developed acute intestinal obstruction due to an internal hernia located between the jejunojejunostomy and the end of the biliopancreatic limb, directly between two jejunal limbs with no mesentery involved. Another seven patients with intermittent colicky abdominal pain, re-explored for the suspicion of internal hernia, were found to also have an open window of the same location apart from a hernia at one of the typical hernia sites. Since this gap is systematically closed during RYGBP, no other patient has been observed with this problem. Even very small defects can lead to the development of internal hernias after RYGBP.
\end{abstract}

A. Paroz $\cdot$ J. M. Calmes $\cdot$ S. Romy $\cdot$ M. Suter

Department of Visceral Surgery, CHUV,

Lausanne, Switzerland

V. Giusti

Division of Endocrinology, Diabetology, and Metabolism, CHUV,

Lausanne, Switzerland

M. Suter $(\bowtie)$

Department of Surgery, Hôpital du Chablais,

1860 Aigle, Switzerland

e-mail: michelsuter@netplus.ch
Patients with suggestive symptoms must be explored. Closure of the jejunojejunal defect with nonabsorbable sutures prevents the development of an internal hernia between the jejunal loops at the jejunojejunostomy.

Keywords Gastric bypass · Laparoscopy · Internal hernia . Complications

\section{Introduction}

Many bariatric surgeons currently consider Roux-en-Y gastric bypass (RYGBP) as the procedure of choice for the surgical treatment of morbid obesity. In carefully selected patients, RYGBP is followed by a very satisfactory weight loss, correction or at least improvement of most obesityrelated comorbidities, and a marked improvement of quality of life. Mineral and vitamin deficiencies are common after RYGBP, but complications requiring interventional therapies are relatively uncommon. The more frequent are stenosis at the gastrojejunostomy, which occurs in up to $5 \%$ of the patients, mostly during the first postoperative semester, and internal hernia $(\mathrm{IH})$ that can develop at any time and affect between 1 and $7.5 \%$ of the patients [1-7]. The laparoscopic approach to RYGBP, currently used by an increasing number of surgeons, has been shown to improve the postoperative course and to reduce the procedure-related morbidity. However, it seems to result in a greater incidence of long-term IH than the traditional open approach [8]. This may be related to the fact that laparoscopy reduces tissue trauma and therefore causes fewer adhesions than laparotomy.

Depending on the surgical technique used for RYGBP, three traditional locations for IH have been described: the 
mesojejunal defect, the Petersen defect, and the mesocolic defect, the latter defect affecting only patients in whom the retrocolic route has been used for the Roux-en-Y jejunal limb. We have reported on IH in our first 607 patients and found that most hernias developed in the mesojejunal window (57\%), followed by the Petersen defect $(27 \%)$ [2]. We also found that systematic closure of all mesenteric defects with running nonabsorbable sutures resulted in a reduced rate of IH compared with absorbable sutures.

The aim of this paper is to report an unusual location of $\mathrm{IH}$ after laparoscopic RYGBP, which developed between the jejunojejunostomy and the extremity of the biliopancreatic limb. We have recently observed ten patients with this location, of whom three presented with acute intestinal obstruction.

\section{Patients and Methods}

We offer RYGBP to morbidly obese patients with a body mass index $(\mathrm{BMI})>40 \mathrm{~kg} / \mathrm{m}^{2}$ and to severely obese patients with a BMI $>35 \mathrm{~kg} / \mathrm{m}^{2}$ with severe comorbidity after complete evaluation by a multidisciplinary team. All the patient data are collected prospectively in a database. Our surgical technique has been described in details elsewhere [9]. Briefly, the operation is performed through six trocars with a $45^{\circ}$ angled optic. The stomach is divided just below the cardia with a linear stapler to form a proximal gastric pouch of approximately $10-15 \mathrm{ml}$. The jejunum is divided about $30 \mathrm{~cm}$ from the angle of Treitz. Using the retrocolic and retrogastric route, we perform the gastrojejunostomy using a $21-\mathrm{mm}$ circular stapler. The jejunojejunal anastomosis is performed with a linear stapler, with a Roux limb of $100 \mathrm{~cm}$ in patients with a BMI up to 48 and $150 \mathrm{~cm}$ if the BMI is higher.

Mesenteric windows were closed with interrupted absorbable sutures initially, since November 2002 with separate stitches of nonabsorbable sutures, and as of March 2004 using running nonabsorbable sutures.

\section{Results}

A total of 1,015 patients underwent laparoscopic RYGBP in our departments between June 1999 and August 2008. Of these, 57 patients $(5.6 \%)$, who developed symptoms sufficiently important to warrant surgical re-exploration, were diagnosed with an IH, including two in the immediate postoperative period. The most common symptom, acute colicky abdominal pain, was present in $47(82,4 \%)$ of these patients. Intestinal obstruction, either complete or partial, developed in 19 (33.3\%) subjects. Of the latter subjects, 11 had experienced acute abdominal cramps one or more times before presenting with obstruction. In two patients, asymptomatic mesenteric defects were found at systematic abdominal exploration during an unrelated procedure.

Except for the two patients who were reoperated early after their primary procedure, the delay between RYGBP and re-exploration varied between 6 and 85 months (mean 30 months), during which a loss of a mean of 14.7 BMI units $(0.4-30)$ was observed. A single defect was found in $30(54.5 \%)$ patients and two or more in the others. The most common defect was at the mesojejunal window in 28 (51\%) patients, followed by the Petersen defect in $22(40 \%)$ and the mesocolon in $18(32 \%)$.

In three patients presenting with acute obstruction, an $\mathrm{IH}$ was found at an unusual location, between the two jejunal loops at the site of the jejunojejunostomy (Figs. 1 and 2). This window developed between the end of the staple line creating the jejunojejunostomy and the running nonabsorbable suture closing the mesenteric window between the afferent limb and the foot of the Roux limb (Fig. 3). Laparoscopy was used as the initial surgical approach in two of them, but conversion was necessary in one, and laparotomy was performed in the last patient. After reduction of the hernia, the bowel proved to be viable in all three patients. The defect was closed using a running nonabsorbable suture, and the postoperative course was uneventful. In seven symptomatic patients reoperated for the suspicion of an $\mathrm{IH}$ and who were found to present a hernia at one of the three common sites, a similar window was found and closed between the two jejunal loops at the jejunojejunostomy. A total of ten $(1 \%)$ of our patients therefore were found with this unusual and so far never described IH.

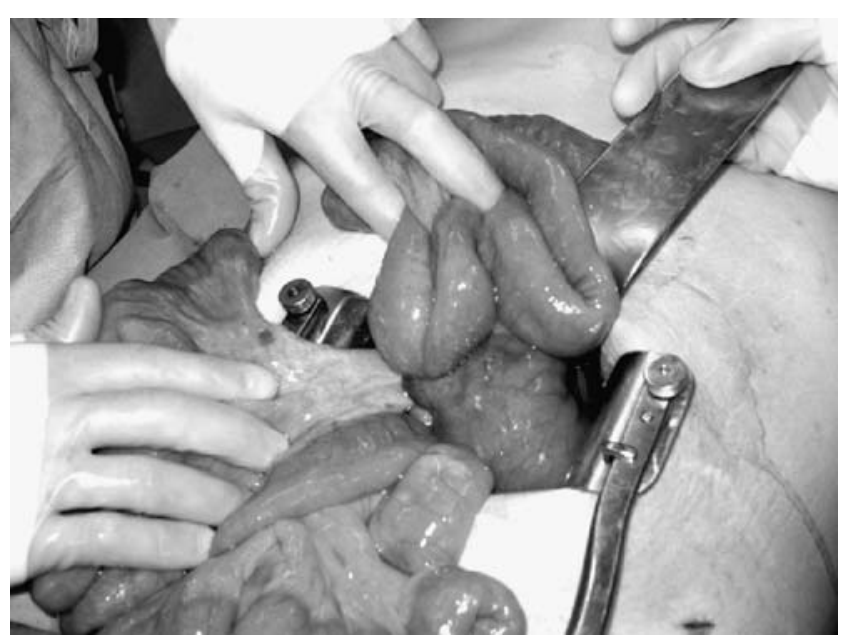

Fig. 1 Internal hernia between the two jejunal loops at the jejunojejunostomy 


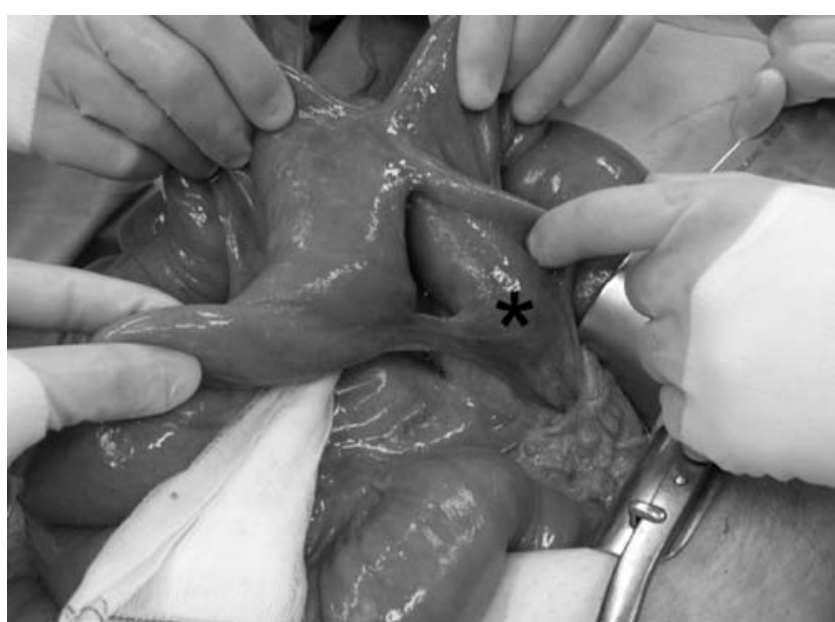

Fig. 2 Gap between the two jejunal loops at the jejunojejunostomy after reduction of the herniated bowel. Asterisk: end of the biliopancreatic limb

\section{Discussion}

The prevalence of IH after RYGBP varies up to $7.5 \%$ in the literature $[1,3-6,8,9]$. In a larger retrospective study encompassing 26 papers reporting on a total of $300 \mathrm{IH}$ after RYGBP, Ianelli et al. found a prevalence of $2.5 \%$ [3]. In our own series totaling 1,015 laparoscopic RYGBP, 57 patients $(5.6 \%)$ developed an IH. Two of these, which developed in the immediate postoperative period through the jejunojejunal mesenteric window that had not been closed during gastric bypass, are the direct consequence of a technical error. In the other patients, IH developed several months or years after RYGBP. Most IH in our experience develop through the jejunojejunal mesenteric window ( $2.7 \%$ of the total patients group), followed by the Petersen window (2.1\%), and the mesocolic window (1.8\%). Since 1999, we have noted a significant decrease in the prevalence of IH in relation to the suturing technique used for closure of the different mesenteric windows. In the first 230 patients, in whom closure was done with absorbable interrupted sutures, 19 (8.2\%) developed an IH. The prevalence was similar (16 patients, 8.8\%) among the next 181 patients in whom nonabsorbable, but still interrupted, sutures were used. Since more than 4 years, running nonabsorbable sutures have been used routinely. This has resulted in a marked drop in the prevalence of $\mathrm{IH}$, with $22(3.6 \%)$ of the last 604 patients affected, a highly significant difference compared with the first two groups $\left(\chi^{2}=10.9, p<0.001\right)$. Despite the fact that most patients with IH develop symptoms within 3 years of surgery, follow-up is shorter for the latter patients, and a few more years are required to confirm these figures.

The new type of IH reported in this paper developed between the biliopancreatic limb and the alimentary limb, just before the jejunojejunostomy. This is clearly related to the technique we used to perform this anastomosis and close the jejunojejunal mesenteric window. First, a small window to allow for the passage of the stapler was made along the mesenteric border of the small bowel. After dividing the jejunum and measuring the length of the alimentary limb, the biliopancreatic limb was brought close to the foot of the latter limb, and both limbs were sutured together using a running nonabsorbable suture, which was continued down the mesenteric window in order to close it. A second suture was placed a few centimeters distally to approximate both limbs and a small opening made on each limb to introduce the linear stapler. After completion of the anastomosis, a small window could remain between the first running suture and the end of the staple line, through which our patients developed their unusual internal hernia (Fig. 3). To the best of our knowledge, this is the first report to describe this unusual location.

Even a very small opening between intestinal loops or in the mesentery can lead to the formation of symptomatic or asymptomatic IH after RYGBP, and small windows may even be more dangerous because they represent a higher risk of incarceration. For mesenteric windows, weight loss probably also contributes, as the thickness of the mesentery decreases, allowing for tiny windows to enlarge progressively. In the case of the jejunojejunal window, however,

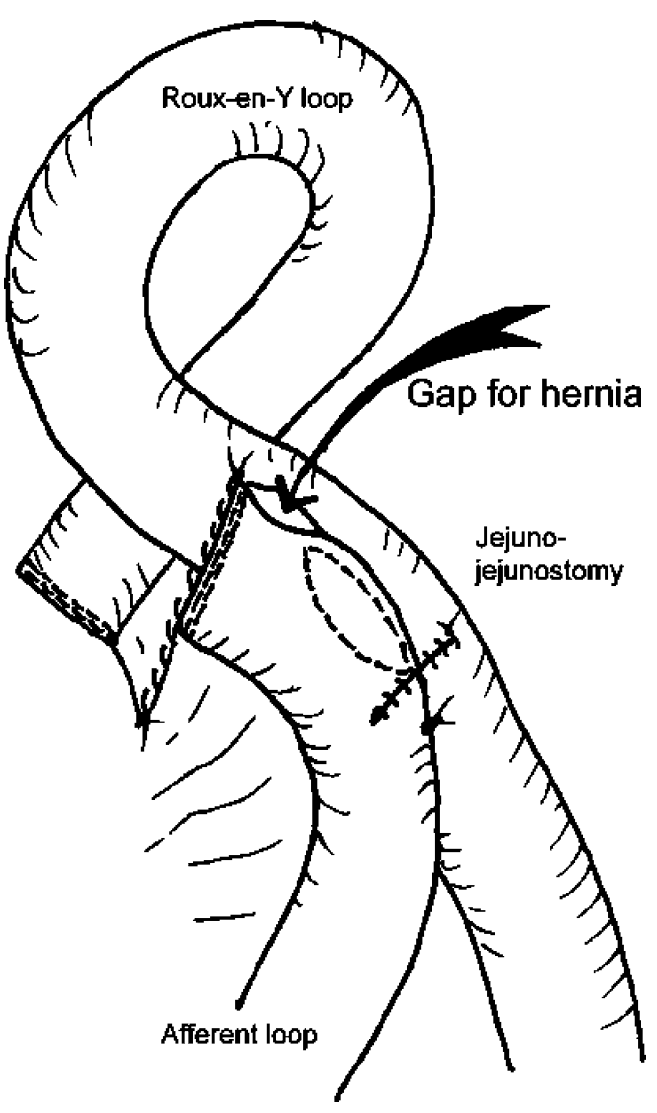

Fig. 3 Schematic drawing of the jejunojejunostomy showing where the new type of hernia reported in this paper developed 
this mechanism is very unlikely to play a role, as the jejunum itself is hardly affected by weight loss. In order to prevent this so far not reported type of $\mathrm{IH}$, we recommend to perform the jejunojejunostomy as close as possible to the distal end of the biliopancreatic limb and to systematically add nonabsorbable sutures in the small gap remaining between the end of the staple line and the suture used to approximate the two jejunal loops and close the mesenteric window. Since our first unusual hernia, we have done this in the last 376 patients, of whom none developed this problem. An alternative strategy would be to start the staple line on the other side, in which case it would be easy to introduce the stapler very close to the first running suture. If an antiobstruction stitch is placed on the other side, however, a small defect can still persist and care must be taken to avoid any potential window, as small as it may be.

IH after RYGBP are often asymptomatic or cause only intermittent symptoms, mostly in the form of intermittent colicky abdominal pain. Intestinal obstruction occurs only in about $33 \%$ of the patients with IH. In the absence of obstruction, diagnosis is difficult, and radiological tests, especially computerized tomography, are often of little help, especially if they are performed during an asymptomatic period. Computed tomography scan can, however, shows small abnormalities suggesting a hernia, like an abnormal course of mesenteric vessels or the "whirl sign" suggestion torsion of a bowel loop. In the presence of suggestive symptoms, a high index of suspicion is the only means to reduce the number of acute complications leading to bowel resection by offering the patient an elective laparoscopic exploration. The cases reported herein show that even very small defects that seem negligible can cause acute symptoms and bowel obstruction with a clear risk of bowel necrosis if prompt surgical treatment is not provided. In order to reduce the risks to develop IH after laparoscopic RYGBP, we recommend careful closure of all the potential defects with running nonabsorbable sutures, including the one between the two jejunal limbs.

\section{References}

1. Ianelli A, Facchiano E, Gugenheim J. Internal hernia after laparoscopic Roux-en-Y gastric bypass for morbid obesity. Obes Surg. 2006;16:1265-71.

2. Paroz A, Calmes JM, Giusti V, et al. Internal hernia after laparoscopic Roux-en-Y gastric bypass for morbid obesity: a continuous challenge in bariatric surgery. Obes Surg. 2005;15:1482-7.

3. Quebbeman BB, Dallai RM. The orientation of the antecolic Roux limb markedly affects the incidence of internal hernias after laparoscopic gastric bypass. Obes Surg. 2005;15:766-70.

4. Fobi MAL, Lee H, Holness R, et al. Gastric bypass operation for obesity. World J Surg. 1998;22:925-35.

5. Podnos YD, Jimenez JC, Wilson SE, et al. Complications after laparoscopic gastric bypass. Arch Surg. 2003;138:957-61.

6. Higa K, Ho T, Boone KB. Internal hernias after laparoscopic Roux-en-Y gastric bypass: incidence, treatment and prevention. Obes Surg. 2003;13:350-4.

7. Comeau E, Gagner M, Inabnet WB, et al. Symptomatic internal hernias after laparoscopic bariatric surgery. Surg Endosc. 2005;19:34-9.

8. Gutt CN, Oniu T, Schemmer P, et al. Fewer adhesions induced by laparoscopic surgery. Surg Endosc. 2004;18:898-906.

9. Suter M, Giusti V, Heraief E, et al. Laparoscopic Roux-en-Y gastric bypass: initial 2-year experience. Surg Endosc. 2003;17:603-9. 